\title{
A note on a determinant identity
}

\section{Antônio Francisco Neto*}

Departamento de Engenharia de Produção, Administração e Economia, Escola de Minas, Campus Morro do Cruzeiro, UFOP, Ouro Preto 35400-000, MG, Brazil

\section{A R T I C L E I N F O}

\section{Keywords:}

Matrix

Determinant

Capelli

\begin{abstract}
A B S T R A C T
In this note we show that the determinant identities obtained by Rezaifar and Rezaee (2007)[1] and Dutta and Pal (2011)-[2] are straightforward consequences of a general result due to Capelli.
\end{abstract}

(C) 2015 Elsevier Inc. All rights reserved.

\section{Introduction}

In two interesting papers [1,2] the authors put forward an identity involving a representation for a determinant. Furthermore, they explore their approach in programming and establish a comparison with other standard existing methods (see, e.g., see section 7 of [1]). Here, our aim is to show that the results of Refs. [1,2] follow directly applying a well known general result due to Capelli (1855-1910) (see Ref. [3] and references therein or Eq. (1) below).

This note is organized as follows. In Section 2, we use the general formula of Capelli to recover the results of Refs. [1,2]. We follow closely Ref. [3]. See also Ref. [4]. In Section 3 we make some concluding remarks.

\section{New proof of the determinant identity}

Let us first give some simple definitions to fix notation. Let $X=\left(x_{i j}\right)$ be an $n \times n$ matrix. We take $I, J \subseteq\{1, \ldots, n\}, I \cup I^{c}=$ $\{1, \ldots, n\}$ and $I \cap I^{c}=\varnothing$. Also, we define $\mathcal{X} \equiv \operatorname{det} X, \partial_{I J}=\left(\partial / \partial x_{i j}\right)=\left(\partial_{i j}\right)=\left(\partial_{i \in I j \in J}\right), X_{I^{c} J^{c}}=\left(x_{i j}\right)=\left(x_{i \in I^{c j} j \in J^{c}}\right), \mathcal{X}_{I^{c} J^{c}}=\operatorname{det} X_{I^{c} J^{c}}$ and $\epsilon(I, J)=(-1)^{\sum_{i \in I} i+\sum_{j \in J} j}$. The following identity is generally attributed to Capelli (see Eq. (1.2) in Ref. [3]):

$$
\operatorname{det}\left(\partial_{I J}\right) \mathcal{X}^{s}=s(s+1) \ldots(s+k-1) \mathcal{X}^{s-1} \epsilon(I, J) \mathcal{X}_{I c J},
$$

with $|I|=k=|J|$. We refer the reader to Ref. [3] for further details towards the proof of this identity and a number of others generalizations, using methods from quantum field theory, like Grassmann-Berezin calculus. The identity in Eq. (1) is our starting point to show that

$$
\mathcal{X}=\frac{1}{\mathcal{X}_{\{1, n\}^{c}\{1, n\}^{c}}} \operatorname{det}\left(\begin{array}{ll}
\mathcal{X}_{\{1\}^{c}\{1\}^{c}} & \mathcal{X}_{\{1\}^{c}\{n\}^{c}} \\
\mathcal{X}_{\{n\}^{c}\{1\}^{c}} & \mathcal{X}_{\{n\}^{c}\{n\}^{c}}
\end{array}\right),
$$

which is equivalent to Eq. (3.1) of Ref. [1] by a suitable adjustment of notation. Indeed, let us take Eq. (1) with $I=J=\{1, n\}$ (then $|I|=|J|=k=2$ ) and $s=2$. Therefore:

$$
\left.\operatorname{det}\left(\partial_{\{1, n\},\{1, n\}}\right) \mathcal{X}^{2}=6 \mathcal{X} \in(\{1, n\},\{1, n\}) \mathcal{X}_{\{1, n\}}\right\}^{c}\{1, n\}^{c},
$$

\footnotetext{
* Tel.: +55 3135591540.

E-mail address: antoniofrancisco1975@gmail.com
} 
where we recall that

$$
\operatorname{det}\left(\partial_{\{1, n\},\{1, n\}}\right)=\operatorname{det}\left(\begin{array}{ll}
\partial_{11} & \partial_{1 n} \\
\partial_{n 1} & \partial_{n n}
\end{array}\right)
$$

and $\mathcal{X}_{\{i\} c\{j\}^{c}}, \mathcal{X}_{\{1, n\}^{c}\{1, n\}^{c}}$ is the determinant of the matrix $(n-1) \times(n-1),(n-2) \times(n-2)$ obtained from $X$ by deleting line $i$ and column $j$, the lines and columns 1 and $n$, respectively. Now observe that $\varepsilon(\{1, n\},\{1, n\})=(-1)^{2(1+n)}=1$, therefore we can write for Eq. (3)

$$
\operatorname{det}\left(\partial_{\{1, n\},\{1, n\}}\right) \mathcal{X}^{2}=6 \mathcal{X} \mathcal{X}_{\{1, n\} c\{1, n\}^{c}} .
$$

A direct calculation gives

$$
\operatorname{det}\left(\partial_{\{1, n\},\{1, n\}}\right) \mathcal{X}^{2}=2 \mathcal{X}\left[\operatorname{det}\left(\partial_{\{1, n\},\{1, n\}}\right) \mathcal{X}\right]+2\left(\partial_{11} \mathcal{X} \partial_{n n} \mathcal{X}-\partial_{1 n} \mathcal{X} \partial_{n 1} \mathcal{X}\right) .
$$

Now we apply Eq. (1) once again, this time with $I=J=\{1, n\}$ (then $|I|=|J|=k=2$ ) and $s=1$ to get

$$
\operatorname{det}\left(\partial_{\{1, n\},\{1, n\}}\right) \mathcal{X}=2 \epsilon(\{1, n\},\{1, n\}) \mathcal{X}_{\{1, n\}^{c}\{1, n\}^{c}}=2 \mathcal{X}_{\{1, n\}^{c}\{1, n\}^{c}} .
$$

Now, taking Eqs. (5), (6) and going back to Eq. (4), observe that

$$
\mathcal{X} \mathcal{X}_{\{1, n\}^{c}\{1, n\}^{c}}=\partial_{11} \mathcal{X} \partial_{n n} \mathcal{X}-\partial_{1 n} \mathcal{X} \partial_{n 1} \mathcal{X}
$$

Using, conveniently, the standard Laplace expansion of the determinant $\mathcal{X}$, we can write

$$
\begin{aligned}
& \mathcal{X}=-\sum_{j}(-1)^{j} x_{1 j} \mathcal{X}_{\{1\}^{c}\{j\}^{c}} \Rightarrow\left\{\begin{array}{l}
\partial_{11} \mathcal{X}=\mathcal{X}_{\{1\}^{c}\{1\}^{c}} \\
\partial_{1 n} \mathcal{X}=(-1)^{1+n} \mathcal{X}_{\{1\}^{c}\{n\}^{c}},
\end{array}\right. \\
& \mathcal{X}=\sum_{j}(-1)^{n+j} x_{n j} \mathcal{X}_{\{n\}^{c}\{j\}^{c}} \Rightarrow\left\{\begin{array}{l}
\partial_{n n} \mathcal{X}=\mathcal{X}_{\{n\}^{c}\{n\}^{c}} \\
\partial_{n 1} \mathcal{X}=(-1)^{1+n} \mathcal{X}_{\{n\}^{c}\{1\}^{c}} .
\end{array}\right.
\end{aligned}
$$

Using the results above in Eq. (7) we have

$$
\begin{aligned}
\mathcal{X X}_{\{1, n\}^{c}\{1, n\}^{c}} & =\mathcal{X}_{\{1\}^{c}\{1\}^{c}} \mathcal{X}_{\{n\}^{c}\{n\}^{c}}-(-1)^{2(1+n)} \mathcal{X}_{\{1\}^{c}\{n\}^{c}} \mathcal{X}_{\{n\}^{c}\{1\}^{c}} \\
& =\mathcal{X}_{\{1\} c}\{1\} c \mathcal{X}_{\{n\}^{c}\{n\}^{c}}-\mathcal{X}_{\{1\}^{c}\{n\}^{c}} \mathcal{X}_{\{n\}^{c}\{1\}^{c}} .
\end{aligned}
$$

and we get Eq. (2) of this note or, equivalently, Eq. (3.1) of Ref. [1]. It is clear from the procedure outlined in this section that the main result stated in Ref. [2], more precisely, Eq. (1) there, is also a consequence of Eq. (1) by taking $I=\{i, j\}=J$ (note that $\varepsilon(\{i, j\}$, $\{i, j\})=1)$.

We close this section by noting that all the results stated here can be alternatively restated entirely in terms of the algebraic/combinatorial framework of Ref. [3]. We will limit ourselves to an indication of the main steps involved. Indeed, Eq. (8) follows from (4) and the following results of Ref. [3]. In what follows all the equations mentioned concern Ref. [3]. First, we take the Grassmann integral representation of $\operatorname{det}\left(\partial_{I J}\right)(\operatorname{det} X)^{s}$ in Eq. (5.13a) with $s=2$ and $I=\{1, n\}=J$ and use Eq. $(4.1)$ to obtain a Grassmann-type representation for $\operatorname{det}\left(X+\bar{\eta} \eta^{T}\right)$, following the notation of Ref. [3]. Next, we use the properties of the Grassmann integral (see Eq. (A.56)) and we observe that the product $\left(\prod \bar{\eta} \eta\right)_{I^{c}, J^{c}}=\bar{\eta}_{2} \eta_{2} \cdots \bar{\eta}_{n-1} \eta_{n-1}$ in the right-hand side of Eq. (5.13a) will select only certain elements of the matrix $\bar{\eta} \eta^{T}$ in the Grassmann (exponential) representation of $\operatorname{det}\left(X+\bar{\eta} \eta^{T}\right)$, i.e., $\bar{\eta}_{1} \eta_{1}, \bar{\eta}_{1} \eta_{n}, \bar{\eta}_{n} \eta_{1}$ and $\bar{\eta}_{n} \eta_{n}$. Finally, the result follows by expanding the exponential representation of $\operatorname{det}\left(X+\bar{\eta} \eta^{T}\right)$ as in Eq. (A.45) and using Eq. (A.95).

\section{Conclusion}

We have shown that the main results of Refs. [1,2] follow directly from an identity of general interest attributed to Capelli. Our result shows the usefulness of Capelli's identity by putting the results of Refs. [1,2] in an unifying perspective. The development of computational procedures might be of interest in order to explore the usefulness of Capelli's identity and further generalizations (see Ref. [3]). Also, it would be interesting to verify if expressions similar to Eq. (8) can be obtained from the other Cayley-type identities introduced in Ref. [3].

\section{Note added in proof}

After the completion of this work we became aware of some previous results related to Refs. [1,2]. See the recently published review article F.F. Abeles, Linear Algebra Appl. 454 (2014) 130-137 and references therein and K. Said, A. Salem, R. Belgacem, A mathematical proof of Dodgson's algorithm, arXiv:0712.0362.

\section{Acknowledgement}

This work was supported by Conselho Nacional de Desenvolvimento Científico e Tecnológico CNPq-Brazil under grants 307824/2009-8 and 454357/2011-7. 


\section{References}

[1] O. Rezaifar, H. Rezaee, Appl. Math. Comp. 188 (2007) 1445-1454.

[2] J. Dutta, S.C. Pal, J. Comp. Math. Sci. 2 (2011) 266-273.

[3] S. Caracciolo, A.D. Sokal, A. Sportiello, Adv. Appl. Math. 50 (2013) 474-594.

[4] A. Abdesselam, Adv. in Appl. Math. 33 (2004) 51-70. 